\title{
New Identifications of the CCH Radical in Planetary Nebulae: A Connection to $\mathrm{C}_{60}$ ?
}

\author{
D. R. Schmidt ${ }^{1,2}$ and L. M. Ziurys ${ }^{1,2,3}$ \\ ${ }^{1}$ Department of Astronomy and Steward Observatory, University of Arizona, 933 N. Cherry Avenue Tucson, AZ 85721-0065, USA \\ ${ }^{2}$ Arizona Radio Observatory, University of Arizona, 933 N. Cherry Avenue Tucson, AZ 85721-0065, USA \\ 3 Department of Chemistry and Biochemistry, University of Arizona, 1305 E. 4th Street Tucson, AZ 85721-0001, USA \\ Received 2017 March 8; revised 2017 August 28; accepted 2017 August 31; published 2017 November 22
}

\begin{abstract}
New detections of $\mathrm{CCH}$ have been made toward nine planetary nebulae (PNe), including K4-47, K3-58, K3-17, M3-28, and M4-14. Measurements of the $N=1 \rightarrow 0$ and $N=3 \rightarrow 2$ transitions of this radical near 87 and $262 \mathrm{GHz}$ were carried out using the new $12 \mathrm{~m}$ and the Sub-Millimeter Telescope (SMT) of the Arizona Radio Observatory (ARO). The presence of fine and/or hyperfine structure in the spectra aided in the identification. $\mathrm{CCH}$ was not observed in two $\mathrm{PNe}$ which are sources of $\mathrm{C}_{60}$. The planetary nebulae with positive detections represent a wide range of ages and morphologies, and all had previously been observed in HCN and HNC. Column densities for $\mathrm{CCH}$ in the $\mathrm{PNe}$, determined from radiative transfer modeling, were $N_{\text {tot }}(\mathrm{CCH}) \sim 0.2-3.3 \times 10^{15} \mathrm{~cm}^{-2}$, corresponding to fractional abundances with respect to $\mathrm{H}_{2}$ of $f \sim 0.2-47 \times 10^{-7}$. The abundance of $\mathrm{CCH}$ was found to not vary significantly with kinematic age across a time span of $\sim 10,000$ years, in contrast to predictions of chemical models. $\mathrm{CCH}$ appears to be a fairly common constituent of $\mathrm{PNe}$ that are carbon-rich, and its distribution may anti-correlate with that of $\mathrm{C}_{60}$. These results suggest that $\mathrm{CCH}$ may be a product of $\mathrm{C}_{60}$ photodestruction, which is known to create $\mathrm{C}_{2}$ units. The molecule may subsequently survive the PN stage and populate diffuse clouds. The distinct, double-horned line profiles for $\mathrm{CCH}$ observed in $\mathrm{K} 3-45$ and $\mathrm{M} 3-28$ indicate the possible presence of a bipolar flow oriented at least partially toward the line of sight.
\end{abstract}

Key words: astrochemistry - ISM: molecules - planetary nebulae: general - radio lines: ISM

\section{Introduction}

Planetary nebulae $(\mathrm{PNe})$ represent the final stage in the evolution of low/intermediate mass $\left(\sim 0.8-8 M_{\text {sol }}\right)$ stars after they transition from the asymptotic giant branch (AGB). Throughout the AGB stage, atomic and molecular material is stripped from the surface of the progenitor star in the form of a superwind, leading to the formation of a circumstellar envelope. The end of the AGB phase is marked by the cessation of mass loss, and the vestigial central star, evolving toward a white dwarf, irradiates the shell of gaseous ejecta, ionizing the surrounding material.

Models have suggested that the bulk of the remnant molecular material should be photodissociated within the first few thousand years of the PN stage (e.g., Redman et al. 2003). This concept seemed to be well-substantiated by observations (e.g., Knapp 1985). Although CO has been identified in numerous PNe (e.g., Huggins et al. 1996, 2005), as well as $\mathrm{H}_{2}$ (e.g., Hora et al. 1999; Bernard-Salas \& Tielens 2005; Likkel et al. 2006; Mata et al. 2016), polyatomic molecules were a rarity. Recent measurements, however, are beginning to change this paradigm. The Helix Nebula, for example, has been found to contain an array of molecules, including $\mathrm{HCN}$, HNC, $\mathrm{HCO}^{+}, \mathrm{H}_{2} \mathrm{CO}, \mathrm{c}_{-} \mathrm{C}_{3} \mathrm{H}_{2}, \mathrm{CCH}$, and $\mathrm{CN}$ (Bachiller et al. 1997; Tenenbaum et al. 2009). Many of these species have been found to be well-distributed throughout the nebula (Zack \& Ziurys 2013; Zeigler et al. 2013; Schmidt \& Ziurys 2017). Such results are particularly surprising, given that the Helix Nebula is one of the oldest PNe known, with an age of $\sim 10,000$ years. $\mathrm{HCN}, \mathrm{HNC}, \mathrm{HCO}^{+}$, and $\mathrm{CN}$ have now been detected toward various planetary nebulae (e.g., Bachiller et al. 1997; Edwards et al. 2014), and observations of the young PNe NGC 7027 and NGC 6537 have revealed a rather surprising molecular inventory, including $\mathrm{N}_{2} \mathrm{H}^{+}, \mathrm{CS}$, and SO (Zhang et al. 2008; Edwards \& Ziurys 2013). Furthermore, in addition to other species, $\mathrm{SiO}$ and $\mathrm{SO}_{2}$ have been recently identified toward the middle-aged PN M2-48 (Edwards \& Ziurys 2014). Perhaps even more remarkable has been the recent discovery of $\mathrm{C}_{60}$ in a few planetary nebulae (e.g., Cami et al. 2010; GarcíaHernández et al. 2010, 2011, 2012; Otsuka et al. 2013). Clearly, the molecular portfolio of planetary nebulae continues to grow.

Very recent work by Schmidt \& Ziurys $(2016,2017)$ has expanded the number of nebulae detected in $\mathrm{HCN}_{1} \mathrm{HCO}^{+}$, and HNC by over a factor of 2, suggesting that these molecules are commonly present in PNe. Furthermore, the sample of 11-13 PNe in which these molecules have been found evenly spans a range of kinematic ages (determined from the intrinsic radius and expansion velocity of the nebula), from $\sim 900$ years to $\sim 11,000$ years, as well as morphologies. Strikingly, the abundances of $\mathrm{HCN}, \mathrm{HNC}$, and $\mathrm{HCO}^{+}$did not appear to vary significantly with kinematic age, in complete contradiction to current chemical modeling (e.g., Redman et al. 2003).

It is naturally of interest to investigate the widespread presence of other polyatomic molecules in PNe. One species of interest is $\mathrm{CCH}$, the ethynyl radical. $\mathrm{CCH}$ is a common constituent of molecular clouds and C-rich circumstellar envelopes (e.g., Zhang et al. 2009; Nishimura et al. 2016), and has also been identified in a few protoplanetary nebulae and toward diffuse clouds (e.g., Lucas \& Liszt 2000; Pardo et al. 2007; Park et al. 2008; Gerin et al. 2011). It has been detected in three PNe to date: NGC 7027, NGC 6537, and the Helix (e.g., Zhang et al. 2008; Tenenbaum et al. 2009; Edwards \& Ziurys 2013).

$\mathrm{CCH}$ is also significant in another aspect: it may be used as a proxy for $\mathrm{C}_{2}$, which cannot be observed in the millimeter regime because it does not have a permanent electric dipole moment. In the laboratory, $\mathrm{C}_{2}$ is thought to be an important building block of $\mathrm{C}_{60}$, which self-assembles through a closed-network mechanism 
Table 1

Target List of Planetary Nebulae

\begin{tabular}{|c|c|c|c|c|c|c|c|}
\hline Source & $\alpha(\mathrm{J} 2000)(\mathrm{h} \mathrm{m} \mathrm{s})$ & $\delta(\mathrm{J} 2000)\left({ }^{\circ} \prime \prime \prime \prime\right)$ & $\theta_{\mathrm{S}}\left({ }^{\prime \prime}\right)$ & Age (years) & Morphology & $\mathrm{C} / \mathrm{O}$ & $\overline{T_{*}\left(10^{3} \mathrm{~K}\right)}$ \\
\hline $\mathrm{Hb} 5$ & $17: 47: 56.2$ & $-29: 59: 41.9$ & 31(a) & $1500_{-540}^{+540}(\mathrm{~b})$ & bipolar & C-rich(c) & $\geqslant 150(d)$ \\
\hline $\mathrm{K} 3-17$ & $18: 56: 18.2$ & $+07: 07: 25.9$ & $8(e)$ & $970_{-300}^{+300}(\mathrm{f})$ & bipolar & C-rich(c), (g) & $\ldots$ \\
\hline K3-45 & $19: 46: 15.6$ & $+24: 11: 03.7$ & $7(\mathrm{~h})$ & $830_{-300}(\mathrm{i})$ & bipolar & $\ldots$ & $\ldots$ \\
\hline $\mathrm{K} 3-58$ & $20: 21: 58.3$ & $+29: 59: 21.1$ & 17(j) & $11070_{-1850}^{+2460}(\mathrm{j})$ & bipolar & $\ldots$ & $\ldots$ \\
\hline M1-7 & $06: 37: 21.0$ & $+24: 00: 36.6$ & 11(e) & $6000_{-1900}^{+1900}(\mathrm{e})$ & bipolar & $1.1(\mathrm{k})$ & 111(1) \\
\hline M3-28 & $18: 32: 41.3$ & $-10: 05: 50.0$ & $17(a)$ & $7700_{-2400}^{+2400}(\mathrm{e})$ & quadrupolar & $\ldots$ & 131(m) \\
\hline M3-55 & $18: 33: 14.8$ & $-10: 15: 19.5$ & 10(j) & $5040_{-2240}^{+1960}(\mathrm{j})$ & bipolar & $\ldots$ & $72(n)$ \\
\hline M4-14 & 19:21:00.7 & $+07: 36: 52.4$ & $15(\mathrm{j})$ & $5550_{-370}^{+3700}(\mathrm{j})$ & quadrupolar & $\ldots$ & $86(\mathrm{~m})$ \\
\hline NGC 2440 & $07: 41: 54.9$ & $-18: 12: 29.7$ & $38(a)$ & $\sim 3400$ (o) & quadrupolar (?) & $1.9(\mathrm{p})$ & $200(p)$ \\
\hline NGC $6772 a(q)$ & $19: 14: 37.3$ & $-02: 42: 19.8$ & $76(a)$ & $10900_{-5200}^{+5200}(\mathrm{r})$ & elliptical & $\ldots$ & $135(\mathrm{~s})$ \\
\hline NGC $6772 b(t)$ & $19: 14: 36.4$ & $-02: 42: 25.0$ & $\cdots$ & $\cdots$ & $\cdots$ & $\cdots$ & $\cdots$ \\
\hline $\mathrm{K} 4-47$ & $04: 20: 45.2$ & $+56: 18: 12.1$ & $8(\mathrm{e})$ & $900_{-500}^{+300}(\mathrm{u})$ & bipolar & C-rich (?) & $115-130(\mathrm{v})$ \\
\hline M1-12 & $07: 19: 21.4$ & $-21: 43: 55.4$ & $2(\mathrm{e})$ & $\sim 1000(\mathrm{w})$ & multipolar & $1.0(\mathrm{x})$ & $29(n)$ \\
\hline M1-20 & $17: 28: 57.6$ & $-19: 15: 53.9$ & 2(a) & $<3900(w)$ & elliptical & $1.2(\mathrm{y})$ & $40-45(z)$ \\
\hline
\end{tabular}

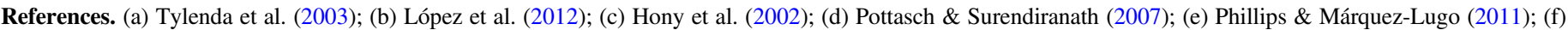

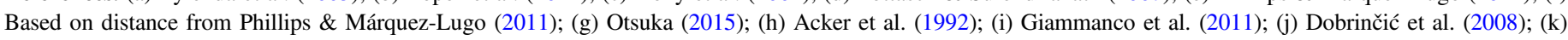

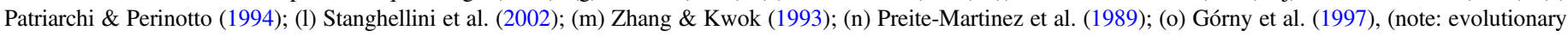

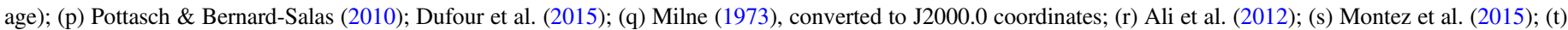

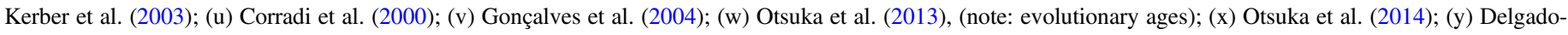
Inglada \& Rodríguez (2014); (z) García-Hernández et al. (2012).

involving $\mathrm{C}$ and $\mathrm{C}_{2}$ (e.g., Dunk et al. 2012). In addition, fragmentation of large carbon clusters shows successive loss of $\mathrm{C}_{2}$ units. Consequently, there may be a correlation or an anticorrelation between $\mathrm{C}_{60}$ and $\mathrm{C}_{2}$, which could be examined through $\mathrm{CCH}$.

Here we report results of a study of $\mathrm{CCH}$ in planetary nebulae. Observations were conducted toward 11 sources from the Schmidt \& Ziurys (2016) survey, as well as two known carriers of $\mathrm{C}_{60} . \mathrm{CCH}$ was newly detected in nine $\mathrm{PNe}$, increasing the number of known $\mathrm{CCH}$-containing nebulae by a factor of 4. From the observations, abundances of this molecule were determined, and correlations with kinematic age and the presence of $\mathrm{C}_{60}$ were examined.

\section{Observations}

The rotational transitions of $\mathrm{CCH}$, a molecule with a ${ }^{2} \Sigma$ ground state, exhibit both fine (spin-rotation) and hyperfine splittings, arising from the unpaired electron $(S=1 / 2)$ and the proton nuclear spin of $I=1 / 2$, respectively. Following a Hund's case $b_{\beta \mathrm{J}}$ coupling scheme, the rotational quantum number is $\mathrm{N}$. The electron spin couples to the rotation to generate a doublet fine structure pattern, labeled by $J$, where $\boldsymbol{J}=\boldsymbol{N}+\boldsymbol{S}$, for every level $N$ except $N=0$. The nuclear spin $I$ then adds to $J$ to create doublet hyperfine levels, indicated by $\mathrm{F}$, where $\boldsymbol{F}=\boldsymbol{J}+\boldsymbol{I}$. The lines of interest for this work are the two hyperfine components of the $N=1 \rightarrow 0, J=3 / 2 \rightarrow 1 / 2$ spin-rotation component at $87,316.9 \mathrm{MHz}(F=2 \rightarrow 1)$ and $87,328.6 \mathrm{MHz}(F=1 \rightarrow 0)$, as well as the $J=7 / 2 \rightarrow 5 / 2$ and $J=5 / 2 \rightarrow 3 / 2$ fine structure components of the $N=3 \rightarrow 2$ transition. Each of the fine structure components of the $N=3 \rightarrow 2$ transition additionally consists of two hyperfine lines: $F=4 \rightarrow 3$ and $3 \rightarrow 2$ and $F=3 \rightarrow 2$ and $2 \rightarrow 1$, respectively. The hyperfine doublets are separated by 2.2-2.5 MHz, or $2.5-2.9 \mathrm{~km} \mathrm{~s}^{-1}$, and are therefore effectively collapsed for the sources studied here. As a consequence, the $N=3 \rightarrow 2$ transition appears as a doublet with lines at $262,005.3 \mathrm{MHz}(J=7 / 2 \rightarrow 5 / 2)$ and $262,066.1 \mathrm{MHz}(J=$
$5 / 2 \rightarrow 3 / 2$ ) - the average frequencies of the collapsed hyperfine doublet. The dipole moment of $\mathrm{CCH}$ is $\sim 0.77 \mathrm{D}$ (Woon 1995).

Observations of the $N=1 \rightarrow 0$ and $N=3 \rightarrow 2$ transitions of $\mathrm{CCH}$ were carried out between 2014 November and 2016 June using the facilities of the Arizona Radio Observatory (ARO). The $12 \mathrm{~m}$ "European" ALMA prototype antenna at Kitt Peak was used to observe the $N=1 \rightarrow 0$ transition, while the ARO $10 \mathrm{~m}$ Sub-Millimeter Telescope (SMT) on Mt. Graham, AZ was employed to measure the $N=3 \rightarrow 2$ lines. The beam size and main beam efficiency are $\theta_{b} \approx 72^{\prime \prime}$ and $\eta_{b} \approx 0.88$ at $87 \mathrm{GHz}$ for the $12 \mathrm{~m}$, and $\theta_{b} \approx 29^{\prime \prime}$ and $\eta_{b} \approx 0.75$ at $262 \mathrm{GHz}$ for the SMT.

The observations performed with the $12 \mathrm{~m}$ telescope utilized a dual-polarization receiver containing ALMA Band 3 sideband separating (SBS) mixers. The image rejection, intrinsic in the mixer architecture, was typically greater than $15 \mathrm{~dB}$. The temperature scale, $T_{A}^{*}$, was determined by the chopper-wheel method. $T_{A}^{*}$ is related to the main beam brightness temperature $\left(T_{R}\right)$ through the equation $T_{R}=T_{A}^{*} / \eta_{b}$, where $\eta_{b}$ is the main beam efficiency. The data were taken using two of three 256channel filter bank backends with $1 \mathrm{MHz}, 250 \mathrm{kHz}$, and $500 \mathrm{kHz}$ resolutions, depending on the source, and the Millimeter Autocorrelator (MAC) with $\sim 781 \mathrm{kHz}$ resolution. All backends were operated in parallel mode to accommodate both receiver channels.

At the SMT, the measurements were conducted with the dual polarization $1.3 \mathrm{~mm}$ receiver, which exploits ALMA Band 6 SBS mixers. Image rejection was generally $>15 \mathrm{~dB}$. The temperature scale, $T_{A}^{*}$, was determined by the standard chopperwheel method, where $T_{R}=T_{A}^{*} / \eta_{b}$. Two filter banks, with $1 \mathrm{MHz}$ and $250 \mathrm{kHz}$ resolution, respectively, were utilized as backends.

The sources searched for $\mathrm{CCH}$ are provided in Table 1, along with their age and size estimates, morphologies, and $\mathrm{C} / \mathrm{O}$ ratios and central star temperatures, if available. As aforementioned, 11 of these sources were previously detected in HCN by 
Schmidt \& Ziurys (2016) and HNC by Schmidt \& Ziurys (2017). All but two of these sources (K3-45 and NGC 6772) were also detected in at least one transition of $\mathrm{HCO}^{+}$. Given the prevalence of carbon-containing molecules in these nebulae, it is likely that they are carbon-rich sources (and indeed some have already been confirmed as such); thus, these $\mathrm{PNe}$ were considered to be good candidates for a search for $\mathrm{CCH}$. Depending on the size of the source relative to the beam, observations were conducted in either position-switching or beam-switching mode. For sources for which position-switching was required, an offset of $+30^{\prime}$ in azimuth was generally used, while a subreflector throw of $\pm 2^{\prime}$ was used for beamswitching. In order to test for possible image contamination, local oscillator shifts were performed for all measurements. Pointing and focus were checked on an hourly basis using nearby strong continuum sources, such as Jupiter or Mars.

\section{Results}

$\mathrm{CCH}$ was detected in 9 of the 11 survey PNe, principally in the $N=3 \rightarrow 2$ transition, which exhibits a "fingerprint" doublet structure separated by $61 \mathrm{MHz}$. In a few sources, the doublet was partially blended together but still discernable. The $N=1 \rightarrow 0$ line, which consists of two well-separated hyperfine components, was only observed in $\mathrm{Hb} 5$ and M3-28; there was significant Galactic contamination in the latter source. $\mathrm{CCH}$ was not detected in NGC 2440 and NGC 6772, as well as either of the two PNe found to contain $\mathrm{C}_{60}$ : M1-12 and M1-20 (García-Hernández et al. 2010). Notably, the nebulae in which $\mathrm{CCH}$ was detected were exclusively bipolar and quadrupolar; observations of the two elliptical PNe in this work, NGC 6772 and M1-20, resulted in non-detections.

The $\mathrm{CCH}$ spectra are presented in Figures 1(a), (b) and 2. The CCH doublet structure is clearly apparent in all spectra, although in some sources it is blended (Hb 5, K3-17). Figure 2 highlights the data from K3-45 and M3-28, and includes the optical images of the two PNe. In these nebulae, the $N=3 \rightarrow 2$ line profiles show distinct red- and blueshifted velocity components.

Table 2 provides the corresponding line parameters for the transitions measured in each source, including the peak antenna temperature $T_{A}^{*}$, LSR velocity, and FWHM line width. The parameters were determined by a Gaussian fit to the line profile for each hyperfine or spin-rotation component. The blended hyperfine structure within each spin-rotation doublet of the $N=3 \rightarrow 2$ transition was modeled as a single feature. For K3-45 and M3-28, the red- and blueshifted velocity components of the $N=3 \rightarrow 2$ spin-rotation doublets were fit individually. As shown in the table, the line intensities fall into the range of $\sim 2-12 \mathrm{mK}$, while the line widths and LSR velocities are comparable to those seen in $\mathrm{HCN}, \mathrm{HNC}$, and $\mathrm{HCO}^{+}$(see Schmidt \& Ziurys 2016, 2017). The individual nebulae are briefly described below.

$\mathrm{Hb}$ 5: Both transitions of $\mathrm{CCH}$ were detected in this hot, young nebula. LSR velocities reside in the range of -2 to $-8.5 \mathrm{~km} \mathrm{~s}^{-1}$ - consistent with those measured for $\mathrm{HCN}$, $\mathrm{HCO}^{+}$, and HNC (Schmidt \& Ziurys 2016, 2017). The narrower line widths of $\sim 5-7 \mathrm{~km} \mathrm{~s}^{-1}$ for the $3 \mathrm{~mm}$ transitions, as opposed to near $40 \mathrm{~km} \mathrm{~s}^{-1}$ for the $N=3 \rightarrow 2$ lines at $1 \mathrm{~mm}$, mimic those observed in the $\mathrm{HCN}$ and $\mathrm{HCO}^{+}$. The difference likely reflects the varying beam sizes coupling to a complex, poly polar source (see López et al. 2012). Note that the prominent bipolar lobes in $\mathrm{Hb} 5$ extend almost an arcminute.
K3-17: Only the $N=3 \rightarrow 2$ component was detected in this PN. The LSR velocity was observed to be $\sim 28 \mathrm{~km} \mathrm{~s}^{-1}$, while the line widths were found to be $\sim 59-66 \mathrm{~km} \mathrm{~s}^{-1}$; the values are consistent with those measured for $\mathrm{HCN}, \mathrm{HCO}^{+}$, and $\mathrm{HNC}$ (Schmidt \& Ziurys 2016, 2017). The CCH line profile, particularly the $J=7 / 2 \rightarrow 5 / 2$ component, may exhibit multiple velocity components. Such velocity features are found in the spectra of $\mathrm{HCN}, \mathrm{HCO}^{+}$, and $\mathrm{HNC}$, and they likely trace the complex filamentary structure of K3-17.

$K 3-45$ : The $N=3 \rightarrow 2$ transition of $\mathrm{CCH}$ detected in this source appears to be double-peaked, particularly in the $J=$ $7 / 2 \rightarrow 5 / 2$ component. The redshifted component lies at a velocity of approximately $45 \mathrm{~km} \mathrm{~s}^{-1}$, while the blueshifted peak is near $20 \mathrm{~km} \mathrm{~s}^{-1}$, on average. These velocities are very close in value to those found in the double-peaked profiles of HCN and HNC (Schmidt \& Ziurys 2016, 2017). Molecular emission is apparently tracing the bipolar outflow in this source.

$K 3-58$ : The $N=3 \rightarrow 2$ transition of $\mathrm{CCH}$ was detected in this small, bipolar nebula. The LSR velocity was observed to be about $21-24 \mathrm{~km} \mathrm{~s}^{-1}$ from the spectrum, with line widths of $\sim 35 \mathrm{~km} \mathrm{~s}^{-1}$. These values are similar to those seen in the HCN, $\mathrm{HNC}$, and $\mathrm{HCO}^{+}$spectra.

M1-7: This PN was detected in the $N=3 \rightarrow 2$ transition of $\mathrm{CCH}$, with an LSR velocity of about $-12 \mathrm{~km} \mathrm{~s}^{-1}$ and line widths of $\sim 33-36 \mathrm{~km} \mathrm{~s}^{-1}$, equivalent to those found for $\mathrm{HCN}$, $\mathrm{HNC}$, and $\mathrm{HCO}^{+}$within the uncertainties. A prominent component near $-34 \mathrm{~km} \mathrm{~s}^{-1}$ can be seen, similar to one observed in HNC.

M3-28: This small, quadrupolar nebula was detected in both transitions of $\mathrm{CCH}$. The $N=1 \rightarrow 0$ transition suffers from Galactic contamination on the redshifted side, obscuring the $F=2 \rightarrow 1$ hyperfine component. The $N=3 \rightarrow 2$ spin-rotation components were found to be U-shaped, with the red- and blueshifted peaks at $V_{\mathrm{LSR}} \sim 43$ and $18 \mathrm{~km} \mathrm{~s}^{-1}$. The redshifted component is about a factor of two broader than its blueshifted counterpart, suggesting some asymmetry in the nebular geometry. Evidence of a $\mathrm{U}$-shape profile was also seen in the $J=3 \rightarrow 2$ spectra of $\mathrm{CO}, \mathrm{HCN}$, and $\mathrm{HCO}^{+}$(Schmidt \& Ziurys 2016), but appears most prominent in $\mathrm{CCH}$.

M3-55: Only the stronger spin component of the $N=3 \rightarrow 2$ transition, $J=7 / 2 \rightarrow 5 / 2$, was clearly detected in this small, bipolar nebula. Estimates of the line parameters are therefore less reliable, but the visible $N=3 \rightarrow 2$ feature indicates $V_{\mathrm{LSR}} \sim 25 \mathrm{~km} \mathrm{~s}^{-1}$ and $\Delta V_{1 / 2} \sim 25 \mathrm{~km} \mathrm{~s}^{-1}$-parameters consistent with those observed for $\mathrm{HCN}, \mathrm{HNC}$, and $\mathrm{HCO}^{+}$, though the line appears to be somewhat wider in $\mathrm{CCH}$.

M4-14: $\mathrm{CCH}$ was detected in the $N=3 \rightarrow 2$ transition in this small, quadrupolar nebula. The measured LSR velocity of $\sim 51 \mathrm{~km} \mathrm{~s}^{-1}$ and $\Delta V_{1 / 2} \sim 33 \mathrm{~km} \mathrm{~s}^{-1}$ for $\mathrm{CCH}$ are analogous to those observed in $\mathrm{HCN}, \mathrm{HCO}^{+}$, and $\mathrm{HNC}$.

K4-47: This young, compact, bipolar nebula was detected in the $N=3 \rightarrow 2$ lines with a velocity of about $-24 \mathrm{~km} \mathrm{~s}^{-1}$ and $\Delta V_{1 / 2}$ of $\sim 38 \mathrm{~km} \mathrm{~s}^{-1}$. These measurements are consistent with data obtained for $\mathrm{HCN}, \mathrm{HNC}$, and $\mathrm{HCO}^{+}$. A "central" component and a weaker, broad "plateau" feature that have been seen in other molecules such as $\mathrm{HCN}$ and $\mathrm{HCO}^{+}$are not apparent in the CCH spectrum (Schmidt \& Ziurys 2016).

\section{Analysis}

$\mathrm{CCH}$ column densities were estimated using the non-LTE radiative transfer code RADEX (Van der Tak et al. 2007). 
胥
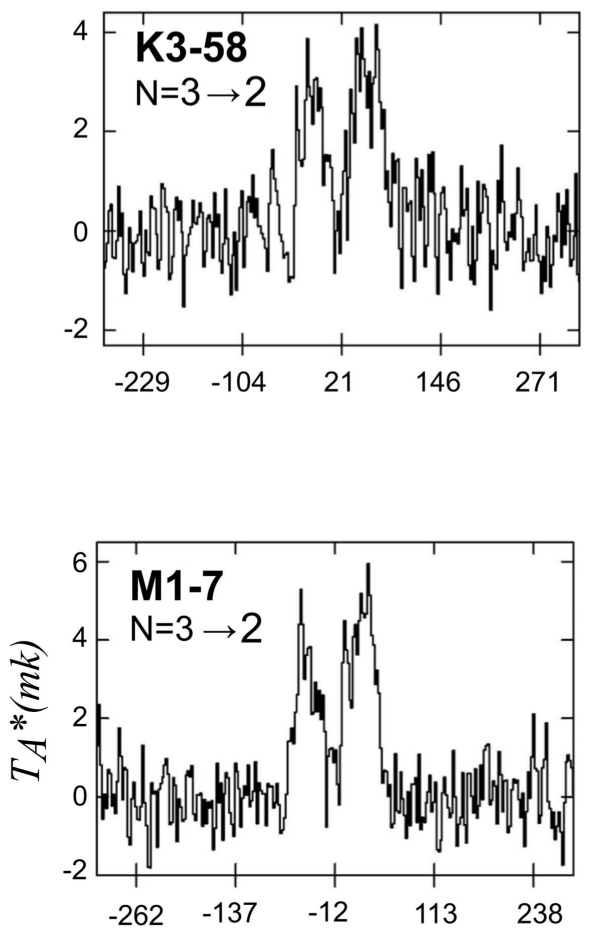
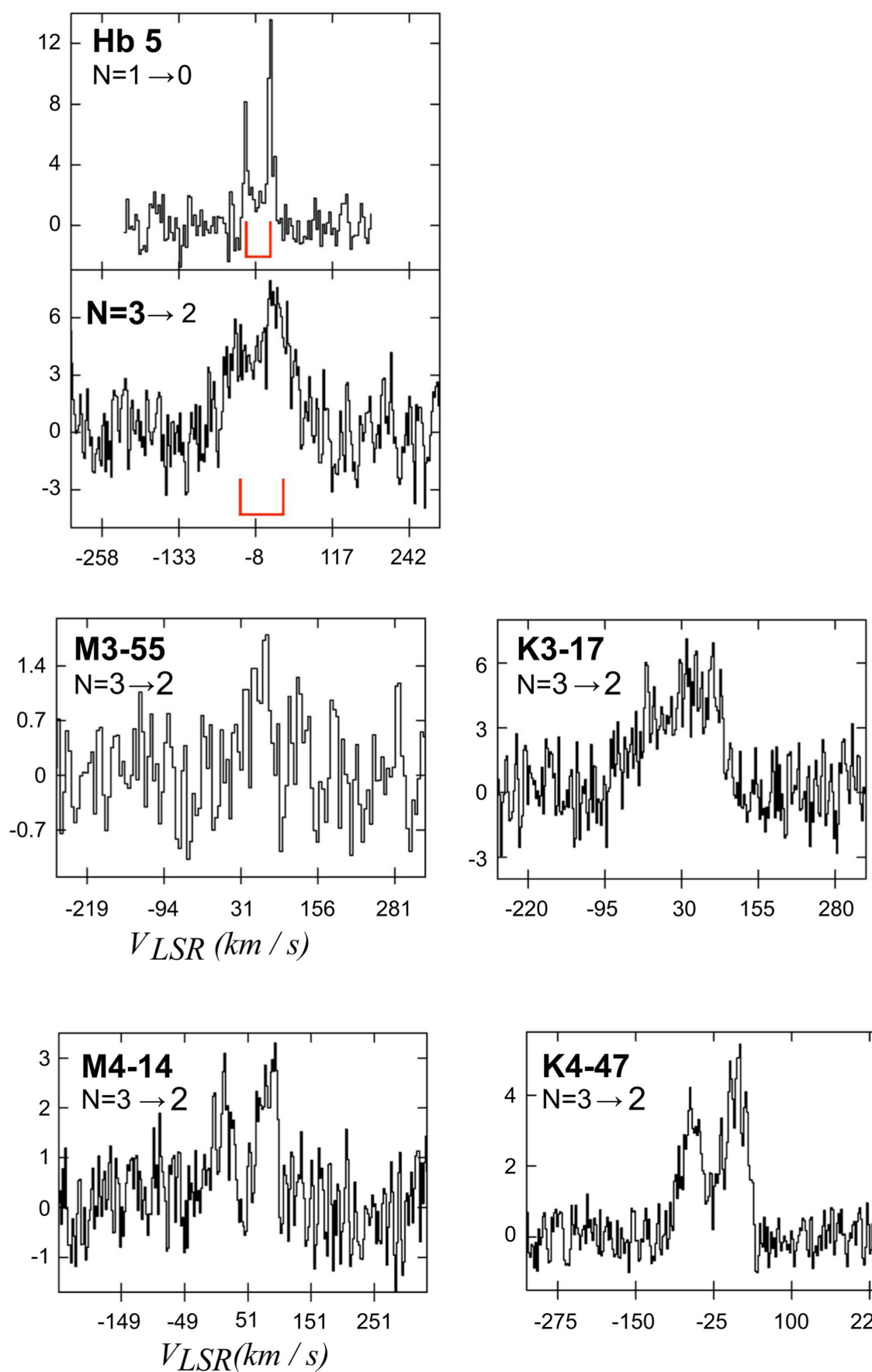

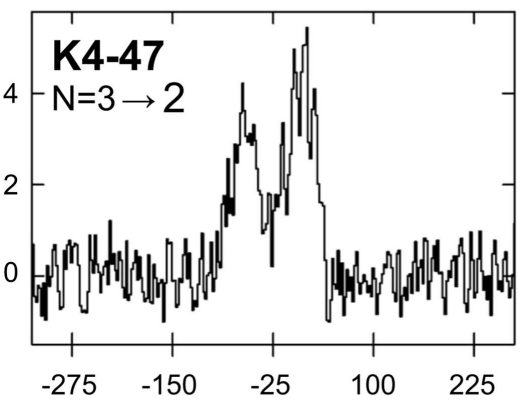

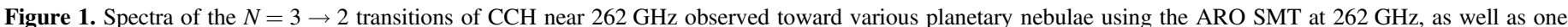

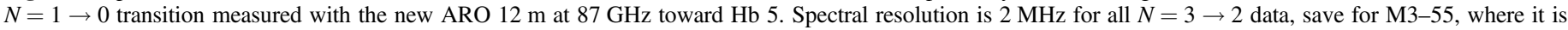

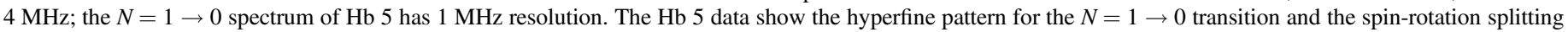

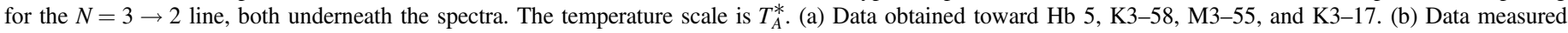
toward M1-7, M4-14, and K4-47.

Briefly, this program predicts line intensities for comparison with those observed by employing the following parameters, which are input by the user: $\mathrm{H}_{2}$ gas density, temperature, and molecular column density. For further details, see Schmidt \& Ziurys (2016). For $\mathrm{CCH}$, both hyperfine and fine structure levels were modeled, based on relative intensities from Ziurys et al. (1982). At most, only two rotational transitions of $\mathrm{CCH}$ were observed for a given source such that at least one variable had to be fixed; in this case, the gas kinetic temperature was held constant at $T_{\mathrm{K}}=20 \mathrm{~K}$, a standard temperature for molecular gas in PNe (see Zack \& Ziurys 2013; Edwards \& Ziurys 2014; Edwards et al. 2014).

For the two sources for which two rotational transitions of $\mathrm{CCH}$ were measured, M3-28 and $\mathrm{Hb} 5$, the $\mathrm{H}_{2}$ density and column density could be adjusted. Specifically, the $\mathrm{H}_{2}$ density was altered between $1.0 \times 10^{4}-1.0 \times 10^{8} \mathrm{~cm}^{-3}$, while the column density was varied between $1.0 \times 10^{11}-1.0 \times 10^{16} \mathrm{~cm}^{-2}$. The range in $\mathrm{H}_{2}$ density was based on the critical densities of the relevant transitions and their Einstein A coefficients for an assumed gas kinetic temperature of $20 \mathrm{~K}$. The densities derived from these 

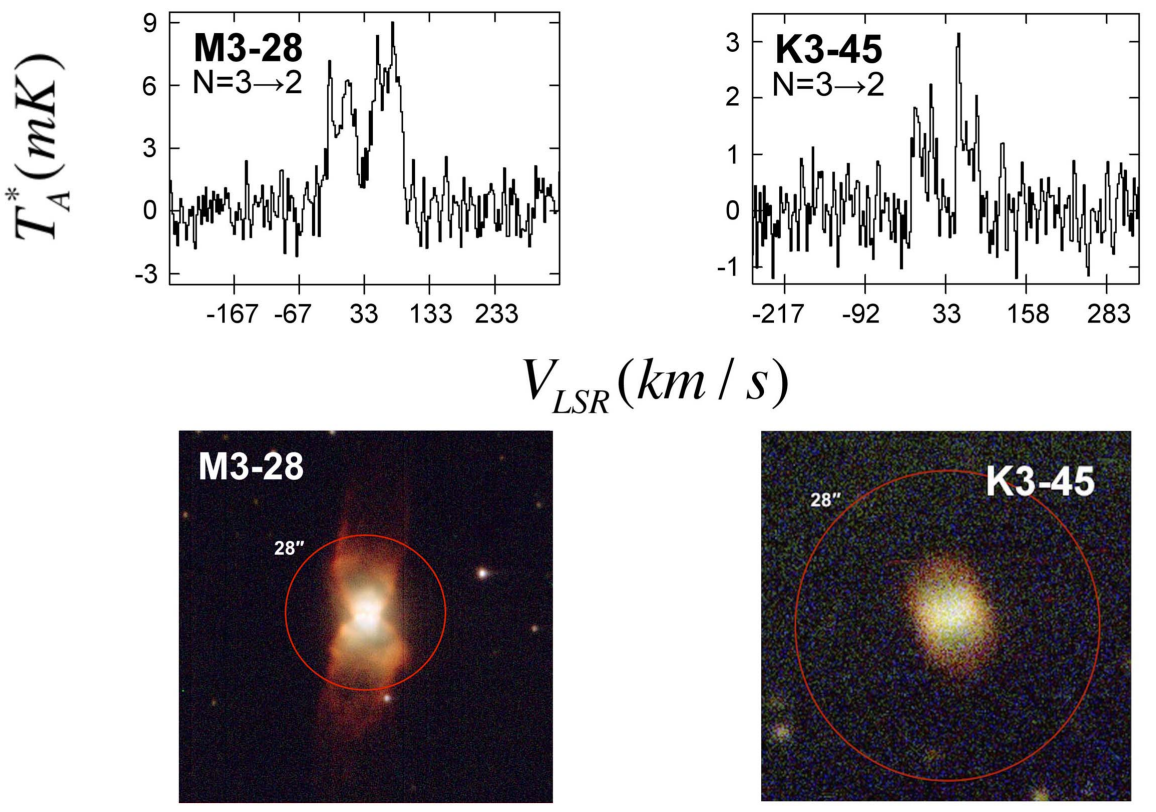

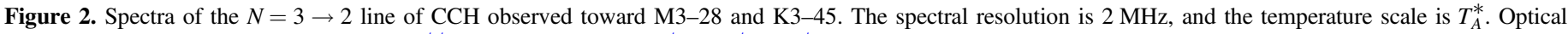

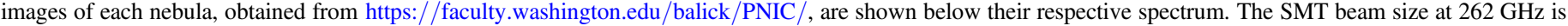

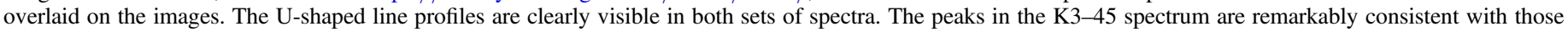
seen in HCN and HNC. These results suggest that both sources contain bipolar outflows oriented partly along the line of sight.

sources fell in the range of $\sim 1-3 \times 10^{5} \mathrm{~cm}^{-3}$, which are consistent with values derived from other molecules in these sources, considering relative dipole moments (e.g., Zack \& Ziurys 2013; Schmidt \& Ziurys 2017). For the remaining sources, the $\mathrm{H}_{2}$ density was fixed to the average of the value for M3-28 and $\mathrm{Hb} 5$. The line width for each fit was set to the average line width for the $\mathrm{CCH}$ transitions observed in that particular source. Additionally, a source filling factor was applied to all line intensities, based on the size of the given nebula from its optical image (see Table 1). Because the molecular gas has a knotty, nonuniform distribution in $\mathrm{PNe}$, a "clump volume filling factor" (the percent of the volume of the nebula filled with clumped material, in this case taken to be the filling factor in the plane of the sky) was also applied to the data. The clump volume filling factors varied from 0.2 to 0.33 , depending on the source (see Schmidt \& Ziurys 2016 for more details). Upon applying these corrections, the resultant column densities were found to lie in the range of $N_{\text {tot }}(\mathrm{CCH}) \sim 0.3-5.8 \times 10^{14} \mathrm{~cm}^{-2}$ when not considering "clumping," and $N_{\text {tot }}(\mathrm{CCH}) \sim 0.2-3.3 \times 10^{15} \mathrm{~cm}^{-2}$ when "clumping" was applied. The non-clump and clump calculations were within a factor of $\sim 3-6$ of each other. The predicted line intensities from the modeling were on average within $20 \%$ of the observed values.

Fractional abundances with respect to $\mathrm{H}_{2}$ were calculated given these column densities. For two sources (K4-47 and $\mathrm{K} 3-58), \mathrm{H}_{2}$ column densities were derived from the measured intensities of the $\nu=1 \rightarrow 0 \mathrm{~S}(1)$ infrared transition at 2.12 microns $\left(8.81 \times 10^{-13} \mathrm{erg} \mathrm{cm}^{-2} \mathrm{~s}^{-1}\right.$ : Lumsden et al. 2001 and $2.27 \times 10^{-12} \mathrm{erg} \mathrm{cm}^{-2} \mathrm{~s}^{-1}$ : Guerrero et al. 2000, respectively). The column density expression of O'Dell et al. (2005) was used, scaled by the appropriate excitation temperature. For K4-47, an excitation temperature of $1550 \mathrm{~K}$ was assumed (Lumsden et al. 2001). As such information was not available for K3-58, the excitation temperature of the Helix ( $~ 900 \mathrm{~K}$; Cox et al. 1998) was used, as the ages of the two nebulae are comparable. This method yielded values of $N_{\text {tot }}\left(\mathrm{H}_{2}\right) \sim 2.9 \times$ $10^{20} \mathrm{~cm}^{-2}$ and $\sim 1.1 \times 10^{20} \mathrm{~cm}^{-2}$ for $\mathrm{K} 4-47$ and $\mathrm{K} 3-58$, respectively, with corresponding uncertainties of $9 \%$ and $30 \%$ (see Schmidt \& Ziurys 2016). For the other sources, $\mathrm{H}_{2}$ column densities were scaled from those of CO (from Schmidt $\&$ Ziurys 2016), assuming $\mathrm{CO} / \mathrm{H}_{2} \sim 10^{-4}$. This ratio is consistent with what is seen in other PNe (see, e.g., Healy \& Huggins 1990; Huggins et al. 2002; Zack \& Ziurys 2013; Edwards et al. 2014). The respective $\mathrm{CO}$ column densities are listed in Table 3 in this work. The derived $\mathrm{H}_{2}$ column densities agree well with those measured in a few PNe based on pure rotational lines of this molecule (Bernard-Salas \& Tielens 2005). Fractional abundances thus derived are $f\left(\mathrm{CCH} / \mathrm{H}_{2}\right) \sim 0.04-3.2 \times 10^{-6}$ in the non-clump case and $f$ $\sim 0.02-4.7 \times 10^{-6}$ with clumping. Overall, M3-28 appears to possess the lowest $\mathrm{CCH}$ abundance while $\mathrm{Hb} 5$ has the highest, with K4-47 following closely behind-similar to what was found for $\mathrm{HNC}$ and $\mathrm{HCN}$ in the same sample of nebulae. Interestingly, M3-28 also exhibits the lowest abundance of $\mathrm{HCO}^{+}$.

\section{Discussion}

\subsection{Evolution of CCH Abundances from CSEs to PNe}

In circumstellar gas, $\mathrm{CCH}$ is believed to be produced through the photodissociation of acetylene near the envelope edge (e.g., Zhang et al. 2008 and references therein):

$$
\mathrm{C}_{2} \mathrm{H}_{2}+h \nu \rightarrow \mathrm{CCH}+\mathrm{H} \text {. }
$$

$\mathrm{CCH}$ is subsequently involved in the formation of long carbon chains and higher order hydrocarbons in the outer envelope (e.g., Mookerjea et al. 2012). Observations have shown that $\mathrm{CCH}$ is indeed a common molecule in the shells of C-rich AGB stars. Abundances for the species in well-known sources of this type such as IRC+10216, CIT6, IRAS 15194-5115, AFGL 4211, and AFGL 3068 fall in the range of $f\left(\mathrm{CCH} / \mathrm{H}_{2}\right) \sim$ 0.24-1.6 $\times 10^{-5}$ (Nyman et al. 1993; Woods et al. 2003; Zhang et al. 2009). From a survey of 35 evolved stars, Fuente 
Table 2

Observations of $\mathrm{CCH}\left(\mathrm{X}^{2} \Sigma^{+}\right)$toward Planetary Nebulae ${ }^{\mathrm{a}}$

\begin{tabular}{|c|c|c|c|c|}
\hline Source & Transition & $\overline{T_{A}^{*}(\mathrm{mK})}$ & $V_{\mathrm{LSR}}\left(\mathrm{km} \mathrm{s}^{-1}\right)$ & $\Delta V_{1 / 2}\left(\mathrm{~km} \mathrm{~s}^{-1}\right)$ \\
\hline \multirow[t]{6}{*}{$\mathrm{Hb} 5$} & $N=1 \rightarrow 0 ; J=3 / 2 \rightarrow 1 / 2$ & & & \\
\hline & $F=2 \rightarrow 1$ & $12.0 \pm 2.0$ & $-3.2 \pm 3.4$ & $7.1 \pm 1.7$ \\
\hline & $F=1 \rightarrow 0$ & $8.0 \pm 2.0$ & $-4.1 \pm 3.4$ & $4.7 \pm 1.7$ \\
\hline & $N=3 \rightarrow 2$ & & & \\
\hline & $J=7 / 2 \rightarrow 5 / 2^{\mathrm{b}, \mathrm{c}}$ & $7.5 \pm 1.0$ & $-8.5 \pm 4.6$ & $42.4 \pm 4.6$ \\
\hline & $J=5 / 2 \rightarrow 3 / 2^{\mathrm{d}, \mathrm{c}}$ & $5.0 \pm 1.0$ & $-2.0 \pm 4.6$ & $42.4 \pm 4.6$ \\
\hline \multirow[t]{4}{*}{ K3-17 } & $N=1 \rightarrow 0$ & $<1$ & $\ldots$ & $\ldots$ \\
\hline & $N=3 \rightarrow 2$ & & & \\
\hline & $J=7 / 2 \rightarrow 5 / 2^{\mathrm{b}, \mathrm{c}}$ & $6.0 \pm 3.0$ & $26.1 \pm 6.9$ & $58.9 \pm 6.9$ \\
\hline & $J=5 / 2 \rightarrow 3 / 2^{\mathrm{d}, \mathrm{c}}$ & $4.0 \pm 3.9$ & $29.6 \pm 6.9$ & $65.9 \pm 6.9$ \\
\hline \multirow[t]{6}{*}{ K3-45 } & $N=1 \rightarrow 0 ; J=3 / 2 \rightarrow 1 / 2$ & $<1$ & $\ldots$ & $\ldots$ \\
\hline & $N=3 \rightarrow 2$ & & & \\
\hline & $J=7 / 2 \rightarrow 5 / 2^{\mathrm{b}, \mathrm{e}}$ & $3.5 \pm 1.0$ & $16.5 \pm 4.6$ & $13.6 \pm 4.6$ \\
\hline & & $2.2 \pm 1.0$ & $45.5 \pm 4.6$ & $13.6 \pm 4.6$ \\
\hline & $J=5 / 2 \rightarrow 3 / 2^{\mathrm{d}, \mathrm{e}}$ & $2.0 \pm 1.0$ & $24.0 \pm 4.6$ & $14.1 \pm 4.6$ \\
\hline & & $2.0 \pm 1.0$ & $46.0 \pm 4.6$ & $11.8 \pm 4.6$ \\
\hline \multirow[t]{4}{*}{ K3-58 } & $N=1 \rightarrow 0 ; J=3 / 2 \rightarrow 1 / 2$ & $<2$ & $\ldots$ & $\ldots$ \\
\hline & $N=3 \rightarrow 2$ & & & \\
\hline & $J=7 / 2 \rightarrow 5 / 2^{\mathrm{b}}$ & $3.4 \pm 1.0$ & $20.5 \pm 4.6$ & $35.3 \pm 4.6$ \\
\hline & $J=5 / 2 \rightarrow 3 / 2^{\mathrm{d}}$ & $2.8 \pm 1.0$ & $24.0 \pm 4.6$ & $35.3 \pm 4.6$ \\
\hline \multirow[t]{3}{*}{ M1-7 } & $\begin{array}{l}N=1 \rightarrow 0 ; J=3 / 2 \rightarrow 1 / 2 \\
N=3 \rightarrow 2\end{array}$ & $<1$ & $\ldots$ & $\ldots$ \\
\hline & $J=7 / 2 \rightarrow 5 / 2^{\mathrm{b}}$ & $5.5 \pm 1.5$ & $-12.5 \pm 4.6$ & $36.0 \pm 4.6$ \\
\hline & $J=5 / 2 \rightarrow 3 / 2^{\mathrm{d}}$ & $4.0 \pm 1.5$ & $-12.0 \pm 4.6$ & $33.0 \pm 4.6$ \\
\hline \multirow[t]{7}{*}{ M3-28 } & $N=1 \rightarrow 0 ; J=3 / 2 \rightarrow 1 / 2$ & & & \\
\hline & $F=1 \rightarrow 0^{f}$ & $1.8 \pm 0.6$ & $\sim 30^{\mathrm{g}}$ & $\sim 30^{\mathrm{g}}$ \\
\hline & $N=3 \rightarrow 2$ & & & \\
\hline & $J=7 / 2 \rightarrow 5 / 2^{\mathrm{b}, \mathrm{e}}$ & $8.5 \pm 2.5$ & $43.5 \pm 4.6$ & $25.9 \pm 4.6$ \\
\hline & & $7.5 \pm 2.5$ & $20.5 \pm 4.6$ & $14.1 \pm 4.6$ \\
\hline & $J=5 / 2 \rightarrow 3 / 2^{\mathrm{d}, \mathrm{e}}$ & $6.0 \pm 2.0$ & $43.0 \pm 4.6$ & $23.5 \pm 4.6$ \\
\hline & & $8.0 \pm 2.0$ & $16.0 \pm 4.6$ & $11.8 \pm 4.6$ \\
\hline \multirow[t]{3}{*}{ M3-55 } & $N=1 \rightarrow 0 ; J=3 / 2 \rightarrow 1 / 2$ & $<2$ & & \\
\hline & $N=3 \rightarrow 2$ & & $\ldots$ & $\ldots$ \\
\hline & $J=7 / 2 \rightarrow 5 / 2^{\mathrm{b}, \mathrm{h}}$ & $1.4 \pm 0.4$ & $25.0 \pm 4.6$ & $25.2 \pm 4.6$ \\
\hline \multirow[t]{4}{*}{ M4-14 } & $N=1 \rightarrow 0 ; J=3 / 2 \rightarrow 1 / 2$ & $<2$ & & \\
\hline & $N=3 \rightarrow 2$ & & $\ldots$ & $\ldots$ \\
\hline & $J=7 / 2 \rightarrow 5 / 2^{\mathrm{b}}$ & $2.6 \pm 1.0$ & $50.5 \pm 4.6$ & $33.0 \pm 4.6$ \\
\hline & $J=5 / 2 \rightarrow 3 / 2^{\mathrm{d}}$ & $2.4 \pm 1.0$ & $51.0 \pm 4.6$ & $33.0 \pm 4.6$ \\
\hline \multirow[t]{4}{*}{ K4-47 } & $N=1 \rightarrow 0 ; J=3 / 2 \rightarrow 1 / 2$ & $<1$ & & \\
\hline & $N=3 \rightarrow 2$ & & & \\
\hline & $J=7 / 2 \rightarrow 5 / 2^{\mathrm{b}}$ & $5.0 \pm 1.0$ & $-26.9 \pm 4.6$ & $37.7 \pm 4.6$ \\
\hline & $J=5 / 2 \rightarrow 3 / 2^{\mathrm{d}}$ & $3.4 \pm 1.0$ & $-20.4 \pm 4.6$ & $37.7 \pm 4.6$ \\
\hline NGC 2440 & $N=1 \rightarrow 0 ; J=3 / 2 \rightarrow 1 / 2$ & $<1$ & $\ldots$ & $\ldots$ \\
\hline NGC $6772 a^{i}$ & $N=1 \rightarrow 0 ; J=3 / 2 \rightarrow 1 / 2$ & $<1$ & $\ldots$ & $\ldots$ \\
\hline \multirow[t]{2}{*}{ NGC $6772 b^{i}$} & $N=1 \rightarrow 0 ; J=3 / 2 \rightarrow 1 / 2$ & $<2$ & $\ldots$ & $\ldots$ \\
\hline & $N=3 \rightarrow 2$ & $<5$ & & \\
\hline M1-12 & $N=3 \rightarrow 2$ & $<4$ & 90.5 & \\
\hline M1-20 & $N=3 \rightarrow 2$ & $<4$ & 70.9 & \\
\hline
\end{tabular}

Notes.

a Measured with $1 \mathrm{MHz}$ resolution unless noted otherwise.

b Blend of $F=4 \rightarrow 3$ and $F=3 \rightarrow 2$ hyperfine components.

c Partially blended spin-rotation components.

d Blend of $F=3 \rightarrow 2$ and $F=2 \rightarrow 1$ hyperfine components.

e U-shaped profile; fit with two velocity components.

${ }^{f}$ Partially obscured by Galactic contamination.

g Blended velocity components.

h Measured with $4 \mathrm{MHz}$ resolution.

${ }^{\mathrm{i}}$ See the text for the source description. 
Table 3

Column Densities and Fractional Abundances

\begin{tabular}{|c|c|c|c|c|c|}
\hline Source & & $N\left(\mathrm{H}_{2}\right)\left(\mathrm{cm}^{-3}\right)$ & $N_{\text {tot }}(\mathrm{CCH})\left(\mathrm{cm}^{-2}\right)$ & $N_{\text {tot }}(\mathrm{CO})\left(\mathrm{cm}^{-2}\right)$ & $\overline{f f\left(\mathrm{CCH} / \mathrm{H}_{2}\right)}$ \\
\hline \multirow[t]{2}{*}{$\mathrm{Hb} 5$} & Clump & $1.4 \mathrm{e} 5$ & $2.0 \pm 0.4 \times 10^{14}$ & $4.3 \pm 1.5 \times 10^{15}$ & $4.7 \pm 0.9 \times 10^{-6}$ \\
\hline & Non-clump & $1.9 \mathrm{e} 5$ & $7.9 \pm 2.1 \times 10^{13}$ & $2.5 \pm 2.4 \times 10^{15}$ & $3.2 \pm 1.6 \times 10^{-6}$ \\
\hline K3-17 & Clump & $1.8 \mathrm{e} 5$ & $3.3 \pm 1.9 \times 10^{15}$ & $3.9 \pm 2.0 \times 10^{17}$ & $8.5 \pm 3.3 \times 10^{-7}$ \\
\hline \multicolumn{6}{|l|}{ K3-45 } \\
\hline \multirow[t]{2}{*}{ Blue-shifted } & Clump & $1.8 \mathrm{e} 5$ & $3.4 \pm 1.9 \times 10^{14}$ & $2.9 \pm 1.6 \times 10^{17}$ & $1.2 \pm 0.5 \times 10^{-7}$ \\
\hline & Non-clump & $2.3 \mathrm{e} 5$ & $9.5 \pm 6.4 \times 10^{13}$ & $6.7 \pm 1.9 \times 10^{16}$ & $1.4 \pm 0.5 \times 10^{-7}$ \\
\hline \multirow[t]{2}{*}{$\mathrm{K} 3-58$} & Clump & $1.8 \mathrm{e} 5$ & $2.6 \pm 0.9 \times 10^{14}$ & $\ldots$ & $2.4 \pm 0.4 \times 10^{-6}$ \\
\hline & Non-clump & $2.3 \mathrm{e} 5$ & $5.5 \pm 2.5 \times 10^{13}$ & $\ldots$ & $5.0 \pm 1.1 \times 10^{-7}$ \\
\hline \multirow[t]{2}{*}{ M1-7 } & Clump & $1.8 \mathrm{e} 5$ & $5.7 \pm 2.0 \times 10^{14}$ & $8.3 \pm 7.4 \times 10^{17}$ & $6.9 \pm 3.3 \times 10^{-8}$ \\
\hline & Non-clump & $2.3 \mathrm{e} 5$ & $1.7 \pm 0.7 \times 10^{14}$ & $1.1 \pm 0.2 \times 10^{17}$ & $1.5 \pm 0.3 \times 10^{-7}$ \\
\hline \multicolumn{6}{|l|}{ M3-28 } \\
\hline Blue-shifted & Clump & $2.1 \mathrm{e} 5$ & $2.2 \pm 1.4 \times 10^{14}$ & $9.3 \pm 7.5 \times 10^{17}$ & $2.4 \pm 1.2 \times 10^{-8}$ \\
\hline & Non-clump & $2.3 \mathrm{e} 5$ & $3.4 \pm 1.7 \times 10^{13}$ & $2.7 \pm 2.1 \times 10^{16}$ & $1.3 \pm 0.6 \times 10^{-7}$ \\
\hline \multirow[t]{2}{*}{ M4-14 } & Clump & $1.8 \mathrm{e} 5$ & $2.4 \pm 1.1 \times 10^{14}$ & $3.6 \pm 1.2 \times 10^{16}$ & $6.7 \pm 1.9 \times 10^{-7}$ \\
\hline & Non-clump & $2.3 \mathrm{e} 5$ & $4.9 \pm 2.7 \times 10^{13}$ & $1.3 \pm 0.8 \times 10^{16}$ & $3.8 \pm 1.6 \times 10^{-7}$ \\
\hline \multirow[t]{2}{*}{ K4-47 } & Clump & $1.8 \mathrm{e} 5$ & $1.0 \pm 0.3 \times 10^{15}$ & $\ldots$ & $3.4 \pm 0.5 \times 10^{-6}$ \\
\hline & Non-clump & $2.3 \mathrm{e} 5$ & $3.0 \pm 1.0 \times 10^{14}$ & $\ldots$ & $1.0 \pm 0.2 \times 10^{-6}$ \\
\hline
\end{tabular}

et al. (1998) found $\mathrm{CCH}$ abundances of $f\left(\mathrm{CCH} / \mathrm{H}_{2}\right) \sim$ $0.025-1.0 \times 10^{-4}$ in $17 \mathrm{C}$-rich stars, with an average value of $\sim 2.7 \times 10^{-5}$.

Studies of $\mathrm{CCH}$ in a limited number of protoplanetary nebulae (PPNe) have also been undertaken, including CRL 2688, CRL 618, and SAO 34504, in which abundances of $\sim 2.0-9.3 \times 10^{-6}$ were reported (Fuente et al. 1998). As far as $\mathrm{PNe}$ are concerned, only a few have been investigated in $\mathrm{CCH}$ prior to this work. NGC 7293 (the Helix Nebula), one of the oldest and most well-studied $\mathrm{PNe}$, was found to have a fractional abundance of $f\left(\mathrm{CCH} / \mathrm{H}_{2}\right) \sim 1 \times 10^{-6}$ at one position within the source (Tenenbaum et al. 2009). In the young planetary nebula NGC 6537 (the Red Spider Nebula), which possesses a hot central star $(\sim 340,000 \mathrm{~K}$ : Matsuura et al. 2005), the $\mathrm{CCH}$ abundance was estimated to be $f$ $\sim 6.9 \times 10^{-8}$ (Edwards \& Ziurys 2013), while another young PN, NGC 7027, was observed to have a value of $f$ $\sim 5.4 \times 10^{-8}$ by Zhang et al. (2008). Our work has expanded the number of PNe detected in $\mathrm{CCH}$ to nine additional nebulae, with abundances in the range of $f \sim 0.02-4.7 \times 10^{-6}$ and an average value of $1.1 \times 10^{-6}$.

Overall, there appears to be a small decrease of a factor of $\sim 5$ in the abundance of $\mathrm{CCH}$ from the AGB to PPN phases. Then, the abundance of the molecule does not appear to significantly change between the PPN and PN stages. These results suggest that, as has been found for HNC (Schmidt \& Ziurys 2017), CCH undergoes some photodestruction in PPNe, but then the abundance "freezes" at the onset of the planetary nebula stage. Therefore, a large fraction of $\mathrm{CCH}$ survives the transition from the AGB stage to the PN stage. This "survival" may result from an even balance between competing production and destruction routes. Based on their models of NGC 7027, Hasegawa \& Kwok (2001) and Zhang et al. (2008) suggest that remnant $\mathrm{CCH}$ from the AGB phase is destroyed, and that the molecule is resynthesized by the ongoing photodestruction of $\mathrm{HCCH}$, as per circumstellar chemistry. As will be discussed, $\mathrm{CCH}$ could also be a product of $\mathrm{C}_{60}$ fragmentation.

\section{2. $\mathrm{CCH}$ in the Late PNe Stage and Beyond}

Few models exist describing the chemistry in PNe. Redman et al. (2003) is one exception, and their model includes $\mathrm{CCH}$. These authors predict a fractional abundance of $\mathrm{CCH}$ (with respect to $\mathrm{H}_{2}$ ) of $1.9 \times 10^{-5}$ at 2550 years, $7.6 \times 10^{-8}$ at 6300 years, and $1.3 \times 10^{-9}$ at 10,050 years - a decline in abundance of about $10^{4}$ over the expected lifetime of PNe ( $~ 10,000$ years). While the abundance around 4000 years is comparable to the average observed values found here, the prediction at 10,050 years is significantly lower. Moreover, as shown in Figure 3, the $\mathrm{CCH}$ abundances of the PNe observed in this work (green circles), as well as those from previous studies (green stars), do not vary significantly with kinematic age. Values span a narrow window of $f \sim 10^{-8}-10^{-6}$ over a period of $\sim 12,000$ years. Most notable are the $\mathrm{CCH}$ abundances for the oldest nebulae, K3-58 ( 11,000 years) and the Helix $(\sim 12,000$ years $)$, which are comparable $\left(2.4 \times 10^{-6}\right.$ and $1 \times 10^{-6}$ ) to those of the younger objects. This is consistent with the observed abundances of $\mathrm{HCN} \mathrm{HCO}^{+}$, and $\mathrm{HNC}$ (also plotted), which are similarly stable over the PN lifetime, though they are lower than those of $\mathrm{CCH}$. Note that some of the ages of the PNe have considerable uncertainties, as listed in Table 1, but the trend nonetheless remains. The estimates of Redman et al. (2003) for $\mathrm{CCH}$ are also plotted with green triangles, showing a steady decline to $f \sim 1 \times 10^{-9}$.

Curiously, $\mathrm{CCH}$ is among the most abundant molecules in the diffuse ISM. Nyman (1984), for instance, searched for the $N=0 \rightarrow 1$ transition in low-density clouds along the lines of sight, detecting the molecule against W49 with an abundance of $f \sim 5.0 \times 10^{-8}$. More recently, Lucas \& Liszt (2000) searched for the $N=0 \rightarrow 1$ line of $\mathrm{CCH}$ in absorption toward 


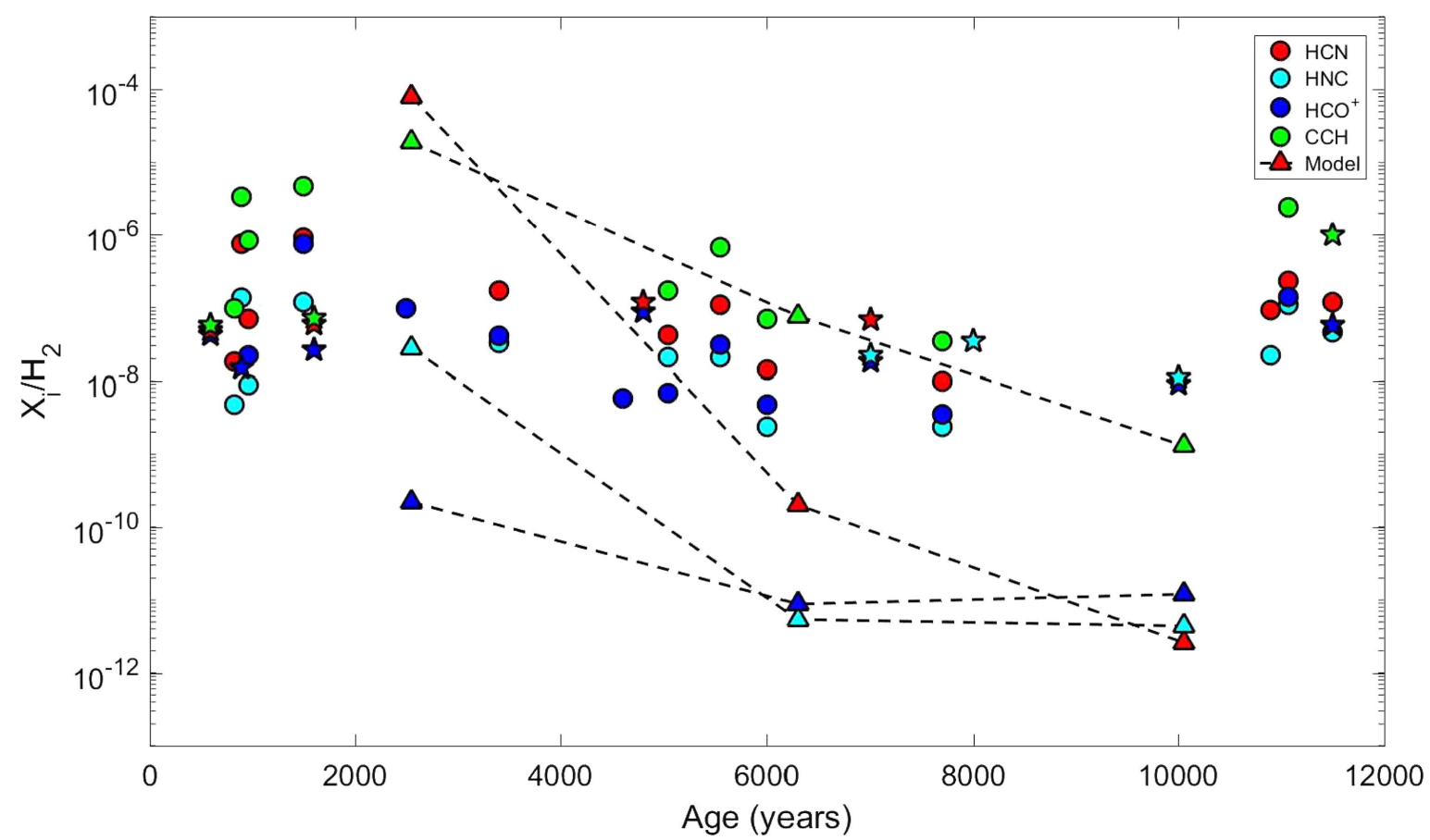

Figure 3. Plot of fractional abundances of $\mathrm{HCN}$ (red), $\mathrm{HCO}^{+}$(blue), $\mathrm{HNC}$ (cyan), and $\mathrm{CCH}$ (green) relative to $\mathrm{H}_{2}$ in PNe vs. kinematic age in years. Circles represent data from this work $(\mathrm{CCH})$ and Schmidt \& Ziurys $\left(2016,2017\right.$ : HCN, $\mathrm{HCO}^{+}$, and $\mathrm{HNC}$ ), while stars indicate measurements for NGC 7027, NGC 6537, M2-48, NGC 7293, NGC 6853, M4-9, NGC 6781, and NGC 6720 from previous studies (Zhang et al. 2008; Tenenbaum et al. 2009; Edwards \& Ziurys 2013; ages from Matsuura et al. 2005; Meaburn et al. 2008; Edwards \& Ziurys 2014; also see Schmidt \& Ziurys 2016, 2017). The red, blue, cyan, and green triangles (with dashed lines) designate the fractional abundances for $\mathrm{HCN}, \mathrm{HCO}^{+}, \mathrm{HNC}$, and $\mathrm{CCH}$, respectively, from the model of Redman et al. (2003). The measured abundances of all molecules appear to stay relatively constant across the $\sim 10,000$ year lifetime of PNe, while the model predicts a significant decrease.

28 background sources in diffuse clouds. These observations revealed fractional abundances with respect to $\mathrm{H}_{2}$ ranging from 1.6-4.2 $\times 10^{-8}$, with a mean abundance of $2.9 \times 10^{-8}$. Additionally, Gerin et al. (2010) observed $\mathrm{CCH}$ along the lines of sight toward three massive star-forming regions, finding a mean fractional abundance of $3.2 \times 10^{-8}$. Lucas \& Liszt (2000) comment that current models do not adequately replicate the abundances reported in their work. Gerin et al. (2011) had more success with their PDR model for CCH, but failed to account for $\mathrm{c}-\mathrm{C}_{3} \mathrm{H}_{2}$.

The abundances measured for PNe in this work are on the order of 1-2 magnitudes higher than those seen in diffuse clouds, as has been found for $\mathrm{HCN}, \mathrm{HNC}$, and $\mathrm{HCO}^{+}$(Schmidt $\&$ Ziurys 2016, 2017). As argued in these prior works, these results are consistent with the notion that the polyatomic molecules seen in the diffuse ISM originate in planetary nebulae. They are ejected in dense globules, which slowly disperse into the surrounding medium.

\section{3. $\mathrm{CCH}$ in Bipolar Nebulae}

$\mathrm{CCH}$ exhibits U-shaped line profiles toward K3-45 and M3-28 in both spin-rotation components (see Figure 2). These profiles suggest the presence of bipolar outflows in these sources oriented at least partially along the line to sight to the observer. Since little kinematic information exists for both of these sources, the $\mathrm{CCH}$ data provides a new window on the morphology of the two objects.

For K3-45, these profiles are duplicated in both $\mathrm{HCN}$ and HNC, with average peak red- and blueshifted velocities of $46.0 \pm 2.2$ and $18.5 \pm 2.2 \mathrm{~km} \mathrm{~s}^{-1}$ (Schmidt \& Ziurys 2016, 2017). These values agree well with those for $\mathrm{CCH}$ in this source: $45-46 \pm 4$ and $16-24 \pm 5 \mathrm{~km} \mathrm{~s}^{-1}$. Furthermore,
Huggins et al. (2005) noted that the nebula exhibited a doublepeaked profile in $\mathrm{CO}$ in the $J=2 \rightarrow 1$ line, overlaid with sharp Galactic emission peaks. The optical picture (Figure 2) shows a blurred image that appears to trace a bipolar flow roughly oriented $\mathrm{N}-\mathrm{S}$, consistent with the position angle of $18^{\circ}$ reported by Corradi et al. (1998), who had first labeled K3-45 as a bipolar nebula from narrowband CCD images. Later, Stanghellini et al. (2002) classified K3-45 as a bipolar nebula based on $\mathrm{H} \alpha$, [O III] $5007 \AA$, and [N II] $6584 \AA$ images, taken from Manchado et al. (1996a). Given the classification for this nebula, our spectra suggest a bipolar flow tilted toward the line of sight, though more data are clearly needed to further investigate the orientation of the flow.

In the case of M3-28, the U-shaped spectrum is seen distinctly in $\mathrm{CCH}$, but is not as obvious in $\mathrm{HCN}$ or $\mathrm{HNC}$. The optical image shows a clear bipolar flow oriented $\mathrm{N}-\mathrm{S}$ (Figure 2) in the plane of the sky. From their morphological survey of northern PNe using two filters corresponding to $\mathrm{H} \alpha$ $+[\mathrm{N} \mathrm{II}]$ and [O III] $5007 \AA$, Manchado et al. (1996b) speculated that this source may be quadrupolar. Thus, $\mathrm{CCH}$ may be tracing a secondary outflow along the line of sight, or the image is simply misleading. The fact that the $\mathrm{HCN}, \mathrm{HNC}$, and $\mathrm{HCO}^{+}$ spectra are not obviously U-shaped may mean that the bipolar flow has lower density and is better traced in $\mathrm{CCH}$.

\subsection{A Connection to $C_{60}$ ?}

The formation mechanisms for $\mathrm{C}_{60}$ in the ISM remain a puzzle. The "bottom-up" scheme, which entails the creation of $\mathrm{C}_{60}$ from smaller compounds such as atomic carbon, $\mathrm{C}_{2}$ units, or possibly smaller fullerenes, is referred to as "closed-network growth," or CNG (e.g., Dunk et al. 2012). However, the timescales necessary for such formation are considered to be 
too long for low-density settings (Micelotta et al. 2012), though Cherchneff et al. (2000) found that such processes are efficient in the envelopes of evolved stars. The proposed "top-down" routes involve the UV photolysis of hydrogenated amorphous carbon (HACs), or UV dehydrogenation of polycyclic aromatic hydrocarbons (PAHs). In the former scheme, hollow structures containing aromatic clusters connected by aliphatic bridging groups are created, which are subsequently dehydrogenated by UV radiation, leading to large fullerene cages that shrink to $\mathrm{C}_{60}$ and $C_{70}$ by releasing $C_{2}$ (Micelotta et al. 2012). Indeed, broad 6-9 $\mu \mathrm{m}$ emission, which may be associated with HACs (García-Hernández et al. 2010, 2011, 2012; Otsuka et al. 2014), was seen in many of the fullerene-containing Magellanic Cloud PNe observed by García-Hernández et al. (2011), as well as in the three PNe studied by Bernard-Salas et al. (2012). Model spectra of $3 \mathrm{~nm}$ HAC particles produced by Bernard-Salas et al. (2012) show that this broad feature can be reproduced relatively successfully, adding credence to this hypothesis. Furthermore, García-Hernández et al. (2011) argue that the presence of PAH clusters, fullerenes, and planar $\mathrm{C}_{24}$ (which they report as having possibly been detected in some of their observed $\mathrm{PNe}$ ) supports the formation of fullerenes from the vaporization of HACs. A possible detection of $\mathrm{C}_{24}$ was also made in the fullerene-containing PN K3-54 (García-Hernández et al. 2012). The latter "top-down" pathway generates graphene, followed by carbon loss and pentagon formation and rearrangement, which then allows the molecule to curl and "stitch" itself up into a closed fullerene (Chuvilin et al. 2010; Berné \& Tielens 2012; Berné et al. 2015). Micelotta et al. (2012) noted, however, that under interstellar conditions, it would be impossible to remove the precise number of carbons necessary to close the graphene flake into a fullerene cage.

Both the "bottom-up" and HAC formation mechanisms for $\mathrm{C}_{60}$ involve $\mathrm{C}_{2}$ as either a precursor or a product of cage shrinkage. Therefore, a relationship may exist between $\mathrm{C}_{60}$ and $\mathrm{C}_{2}$. As discussed, $\mathrm{CCH}$ is the millimeter proxy for $\mathrm{C}_{2}$. The observations conducted here identified $\mathrm{CCH}$ in nine $\mathrm{PNe}$, but failed to detect the molecule in M1-12 and M1-20-two nebulae known to contain $\mathrm{C}_{60}$. While these are small number statistics, the data may suggest an anti-correlation between $\mathrm{C}_{60}$ and $\mathrm{CCH}$.

This apparent lack of correlation may be related to the temperature of the central star. García-Hernández et al. (2012) noted that the stellar temperatures for all $\mathrm{C}_{60}$-containing $\mathrm{PNe}$ fell within a narrow range of $T_{*} \sim 30,000-45,000 \mathrm{~K}$. All of the $\mathrm{PNe}$ in which $\mathrm{CCH}$ was detected for which such information was available display significantly higher stellar temperatures than any of the fullerene sources, within the range of 72,200 K and $\sim 131,000 \mathrm{~K}$ (see Table 1). NGC 2440 and NGC 6772 also have high central star temperatures, though $\mathrm{CCH}$ was not detected in either object. However, these are more complex sources and may not couple well to the telescope beam (see Schmidt \& Ziurys 2016 for optical images and beam sizes at the $\mathrm{CCH}$ frequencies). In the case of NGC 6772, the distribution of $\mathrm{H}_{2}$ is a shell around the central star (MarquezLugo et al. 2013); our observations were centered on the hole in this shell, and thus are not optimized. For NGC 2440, $\mathrm{H}_{2}$ is located in a $10^{\prime \prime}$ region centered on the nebula, and has linear structures extending from this central source (Latter et al. 1995). These structures do not fully fill our $30^{\prime \prime}$ beam, and therefore, beam dilution may be significant. Generally, these data suggest that an increased $T_{*}$ (and elevated UV flux) may lead to the photodissociation of $\mathrm{C}_{60}$. The subsequent release of $\mathrm{C}_{2}$ molecules may then produce $\mathrm{CCH}$ through reactions with $\mathrm{H}_{2}$. There is a substantial activation energy for ejection of $\mathrm{C}_{2}$ from $\mathrm{C}_{60}$ (e.g., Zettergren et al. 2010; Micelotta et al. 2012), but at sufficiently high stellar temperatures, this energy barrier could be overcome. Clearly $\mathrm{H}_{2}$ has to be present for $\mathrm{CCH}$ to form, however, via the proposed process. In both M1-12 and M1-20, $\mathrm{H}_{2}$ was only tentatively detected (Lumsden et al. 2001), although the $J=1 \rightarrow 0$ transition of $\mathrm{CO}$ was apparently observed in the former source (Zhang et al. 2000). Additional observations of $\mathrm{H}_{2}$ and related theoretical work are clearly needed to further examine this prospect.

Alternatively, nondetection of $\mathrm{CCH}$ in these nebulae could result from the large distances to these $\mathrm{PNe}(3.9 \mathrm{kpc}$ and $7.3 \mathrm{kpc}$, respectively; Otsuka et al. 2014) and the lack of sensitivity rather than the absence of $\mathrm{CCH}$ in either source. Using the upper limits for the peak line temperatures of the $N=3 \rightarrow 2$ transitions and the line width for M1-12 from Zhang et al. (2000), an upper limit column density of $4.4 \times 10^{15} \mathrm{~cm}^{-2}$ (considering clumping) was calculated for both sources. Since neither $\mathrm{CO}$ column densities nor $\mathrm{H}_{2}$ brightnesses were available for either source, the average $\mathrm{H}_{2}$ column density measured for K4-47 and K3-58 was used to calculate abundances. This average value is consistent with other measured $\mathrm{H}_{2}$ column densities (Bernard-Salas \& Tielens 2005), and is likely a representative number. An upper limit abundance of $2.2 \times 10^{-5}$ was then obtained, comparable to the abundance prediction of Redman et al. (2003) for 2550 years, the closest point to either nebula's kinematical age.

\section{Conclusions}

The detection of $\mathrm{CCH}$ in nine new planetary nebulae suggests that yet another polyatomic molecule is a fairly common constituent in these objects. The total number of $\mathrm{CCH}$-bearing $\mathrm{PNe}$ is now 12, several of which are quite evolved. $\mathrm{CCH}$ abundances with respect to $\mathrm{H}_{2}$ were seen not to vary with kinematic age, as is also the case for $\mathrm{HCN}, \mathrm{HNC}$, and $\mathrm{HCO}^{+}$. The molecule therefore maintains a high abundance from the AGB stage to the late PN phase, and may populate the diffuse ISM. Furthermore, $\mathrm{CCH}$ is present in those sources that contain $\mathrm{HCN}, \mathrm{HNC}$, and $\mathrm{HCO}^{+}-$an indicator that these particular nebulae are carbon-rich. The radical may be associated with nebulae containing hot central stars, and be linked to the photodestruction of $\mathrm{C}_{60}$ through the intermediate $\mathrm{C}_{2}$. More data is needed to support this hypothesis.

This research was supported by NSF grants AST-1515568 and AST-1140030. The Kitt Peak $12 \mathrm{~m}$ and the SMT are operated by the Arizona Radio Observatory (ARO), with partial support through the NSF University Radio Observatories (URO) program (AST-1140030).

\section{References}

Acker, A., Marcout, J., Ochsenbein, F., Stenholm, B., \& Tylenda, R. 1992, The Strasbourg-ESO Catalogue of Galactic Planetary Nebulae. Parts 1 and 2 (Garching, Germany: European Southern Observatory)

Ali, A., Sabin, L., Snaid, S., \& Basurah, H. M. 2012, A\&A, 541, A98

Bachiller, R., Forveille, T., Huggins, P. J., \& Cox, P. 1997, A\&A, 324, 1123 Bernard-Salas, J., Cami, J., Peeters, E., et al. 2012, ApJ, 757, 41

Bernard-Salas, J., \& Tielens, A. G. G. M. 2005, A\&A, 431, 523

Berné, O., Montillaud, J., \& Joblin, C. 2015, A\&A, 577, A133

Berné, O., \& Tielens, A. G. G. M. 2012, PNAS, 109, 401

Cami, J., Bernard-Salas, J., Peeters, E., \& Malek, S. E. 2010, Sci, 329, 1180 
Cherchneff, I., Le Teuff, Y. H., Williams, P. M., \& Tielens, A. G. G. M. 2000, A\&A, 357, 572

Chuvilin, A., Kaiser, U., Bichoutskaia, E., Besley, N. A., \& Khlobystov, A. N. 2010, NatCh, 2, 450

Corradi, R. L. M., Aznar, R., \& Mampaso, A. 1998, MNRAS, 297, 617

Corradi, R. L. M., Gonçalves, R., Villaver, E., et al. 2000, ApJ, 535, 823

Cox, P., Boulanger, F., Huggins, P. J., et al. 1998, ApJL, 495, L23

Delgado-Inglada, G., \& Rodríguez, M. 2014, ApJ, 784, 173

Dobrinčić, M., Villaver, E., Guerrero, M. A., \& Manchado, A. 2008, AJ, 135, 2199

Dufour, R. J., Kwitter, K. B., Henry, R. B. C., et al. 2015, ApJ, 803, 23

Dunk, P. W., Kaiser, N. K., Hendrickson, C. L., et al. 2012, NatCo, 3, 855

Edwards, J. L., Cox, E. G., \& Ziurys, L. M. 2014, ApJ, 791, 79

Edwards, J. L., \& Ziurys, L. M. 2013, ApJL, 770, L5

Edwards, J. L., \& Ziurys, L. M. 2014, ApJL, 794, L27

Fuente, A., Cernicharo, J., \& Omont, A. 1998, A\&A, 330, 232

García-Hernández, D. A., Iglesias-Groth, S., Acosta-Pulido, J. A., et al. 2011, ApJL, 737, L30

García-Hernández, D. A., Manchado, A., García-Lario, P., et al. 2010, ApJL, 724, L39

García-Hernández, D. A., Villaver, E., García-Lario, P., et al. 2012, ApJ, 760, 107

Gerin, M., De Luca, M., Goicoechea, J. R., et al. 2010, A\&A, 521, L16

Gerin, M., Kaźmierczak, M., Jastrzebska, M., et al. 2011, A\&A, 525, A116

Giammanco, C., Sale, S. E., Corradi, R. L. M., et al. 2011, A\&A, 525, A58

Gonçalves, D. R., Mampaso, A., Corradi, R. L. M., et al. 2004, MNRAS, 355,37

Górny, S. K., Stasiñska, G., \& Tylenda, R. 1997, A\&A, 318, 256

Guerrero, M. A., Villaver, E., Manchado, A., Garcia-Lario, P., \& Prada, F. 2000, ApJS, 127, 125

Hasegawa, T. I., \& Kwok, S. 2001, ApJ, 562, 824

Healy, A. P., \& Huggins, P. J. 1990, AJ, 100, 511

Hony, S., Waters, L. B. F. M., \& Tielens, A. G. G. M. 2002, A\&A, 390, 533

Hora, J. L., Latter, W. B., \& Deutsch, L. K. 1999, ApJS, 124, 195

Huggins, P. J., Bachiller, R., Planesas, P., Forveille, T., \& Cox, P. 2005, ApJS, 160,272

Huggins, P. J., Baschiller, R., Cox, P., \& Forveille, T. 1996, A\&A, 315, 284

Huggins, P. J., Forveille, T., Bachiller, R., et al. 2002, ApJL, 573, L55

Kerber, F., Mignani, R. P., Guglielmetti, F., \& Wiecenec, A. 2003, A\&A, 408, 1029

Knapp, G. R. 1985, in Mass Loss from Red Giants, ed. M. Morris \& B. Zuckerman (Dordrecht: Reidel), 171

Latter, W. B., Kelly, D. M., Hora, J. L., \& Deutsch, L. K. 1995, ApJS, 100, 159

Likkel, L., Dinerstein, H. L., Lester, D. F., Kindt, A., \& Bartig, K. 2006, AJ, 131,1515

López, J. A., Garciá-Diáz, M. T., Steffen, W., Riesgo, H., \& Richer, M. G. 2012, ApJ, 750, 131

Lucas, R., \& Liszt, H. S. 2000, A\&A, 358, 1069

Lumsden, S. L., Puxley, P. J., \& Hoare, M. G. 2001, MNRAS, 328, 419

Manchado, A., Guerrero, M., Stanghellini, L., \& Serra-Ricart, M. 1996a, The IAU Morphological Catalog of Northern Galactic Planetary Nebulae (La Laguna: IAC)
Manchado, A., Stanghellini, L., \& Guerrero, M. A. 1996b, ApJ, 466, L95 Marquez-Lugo, R. A., Ramos-Larios, G., Guerrero, M. A., \& Vázquez, R. 2013, MNRAS, 429, 973

Mata, H., Ramos-Larios, G., Guerrero, M. A., et al. 2016, MNRAS, 459, 841

Matsuura, M., Zijlstra, A. A., Gray, M. D., Molster, F. J., \& Waters, L. B. F. M. 2005, MNRAS, 363, 628

Meaburn, J., López, J. A., \& Richer, M. G. 2008, MNRAS, 384, 497

Micelotta, E. R., Jones, A. P., Cami, J., et al. 2012, ApJ, 761, 35

Milne, D. K. 1973, AJ, 78, 239

Montez, R., Kastner, J. H., Balick, B., et al. 2015, ApJ, 800, 8

Mookerjea, B., Hassel, G. E., Gerin, M., et al. 2012, A\&A, 546, A75

Nishimura, Y., Shimonishi, T., Watanabe, Y., et al. 2016, ApJ, 829, 94

Nyman, L.-A, Olofsson, H., Johansson, L. E. B., et al. 1993, A\&A, 269, 377

Nyman, L.-Å 1984, A\&A, 141, 323

O'Dell, C. R., Henney, W. J., \& Ferland, G. J. 2005, AJ, 130, 172

Otsuka, M. 2015, MNRAS, 452, 4070

Otsuka, M., Kemper, F., Cami, J., Peeters, E., \& Bernard-Salas, J. 2014, MNRAS, 437, 2577

Otsuka, M., Kemper, F., Hyung, S., et al. 2013, ApJ, 764, 77

Pardo, J. R., Cernicharo, J., \& Goicoechea, J. R. 2007, ApJ, 661, 250

Park, J. A., Cho, S.-H., Chang, W. L., \& Yang, J. 2008, AJ, 136, 2350

Patriarchi, P., \& Perinotto, M. 1994, A\&A, 287, 585

Phillips, J. P., \& Márquez-Lugo, R. A. 2011, RMxAA, 47, 83

Pottasch, S. R., \& Bernard-Salas, J. 2010, A\&A, 517, A95

Pottasch, S. R., \& Surendiranath, R. 2007, A\&A, 462, 179

Preite-Martinez, A., Acker, A., Köppen, J., \& Stenholm, B. 1989, A\&AS, 81, 309

Redman, M. P, Viti, S., Cau, P., \& Williams, D. A. 2003, MNRAS, 345, 1291

Schmidt, D. R., \& Ziurys, L. M. 2016, ApJ, 817, 175

Schmidt, D. R., \& Ziurys, L. M. 2017, ApJ, 835, 79

Stanghellini, L., Villaver, E., Manchado, A., \& Guerrero, M. A. 2002, ApJ, 576, 285

Tenenbaum, E. D., Milam, S. N., Woolf, N. J., \& Ziurys, L. M. 2009, ApJL, 704, L108

Tylenda, R., Siódmiak, N., Górny, S. K., Corradi, R. L. M., \& Schwarz, H. E. 2003, A\&A, 405, 627

Van der Tak, F. F. S., Black, J. H., Schöier, F. L., Jansen, D. J., \& van Dishoeck, E. F. 2007, A\&A, 468, 627

Woods, P. M., Schöier, F. L., Nyman, L.-Å, \& Olofsson, H. 2003, A\&A, 402, 617

Woon, D. E. 1995, CPL, 244, 45

Zack, L. N., \& Ziurys, L. M. 2013, ApJ, 765, 112

Zeigler, N., Zack, L. N., Woolf, N. J., \& Ziurys, L. M. 2013, ApJ, 778, 16

Zettergren, H., Johansson, H. A. B., Schmidt, H. T., et al. 2010, JChPh, 133, 104301

Zhang, C. Y., \& Kwok, S. 1993, ApJS, 88, 137

Zhang, H., Sun, J., \& Ping, J. 2000, ChA\&A, 24, 309

Zhang, Y., Kwok, S., \& Trung, D.-V. 2008, ApJ, 678, 328

Zhang, Y., Kwok, S., \& Trung, D.-V. 2009, ApJ, 691, 1660

Ziurys, L. M., Saykally, R. J., Plambeck, R. L., \& Erickson, N. R. 1982, ApJ, 254, 94 